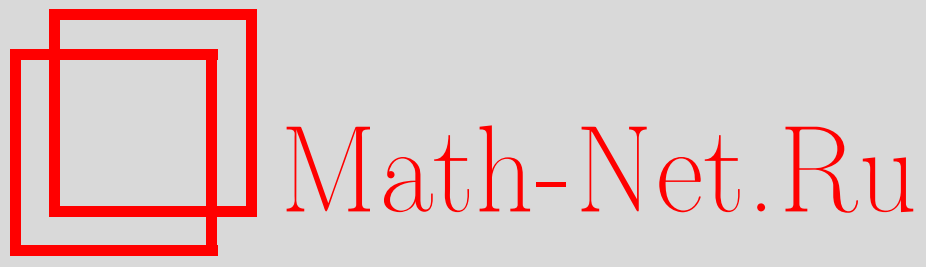

М. В. Павлов, С. П. Царев, Тригамильтоновы структуры егоровских систем гидродинамического типа, Функи. анализ и его прил., 2003, том 37, выпуск $1,38-54$

DOI: https://doi.org/10.4213/faa135

Использование Общероссийского математического портала MathNet.Ru подразумевает, что вы прочитали и согласны с пользовательским соглашением

http://www.mathnet.ru/rus/agreement

Параметры загрузки:

IP : 54.172 .240 .79

26 апреля 2023 г., $11: 19: 40$

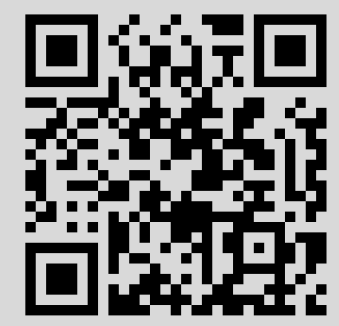




\title{
Тригамильтоновы структуры егоровских систем гидродинамического типа*
}

\author{
(C) 2003. М. В. ПАвЛов, С. П. ЦАРЕв
}

\section{§1. Введение}

Изучение гамильтоновых структур общего вида для систем гидродинамического типа, т. е. систем вида

$$
u_{t}^{i}=\sum_{k=1}^{N} v_{k}^{i}(u) u_{x}^{k}, \quad i=1, \ldots, N,
$$

было начато в работе Дубровина и Новикова [2] и продолжено в работе Мохова и Ферапонтова [5]. В данной работе мы доказываем теорему о наличии трех гамильтоновых структур для диагонализируемой гамильтоновой системы гидродинамического типа (1), которая имеет две физические симметрии: инвариантность относительно преобразования Галилея и относительно растяжения, а также обладает еще рядом свойств - егоровостью метрики гамильтоновой структуры и полупростотой матрицы $\left(\left(r^{i}-r^{k}\right) \beta_{i k}\right)$ (см. ниже $\left.\$ 4\right)$. Известно достаточно много физически интересных систем гидродинамического типа такого вида: усредненные уравнения на $N$-фазных решениях уравнения Кортевега-де Фриза (уравнения Уизема) и нелинейного уравнения Шрёдингера, а также бездисперсионные пределы векторного нелинейного уравнения Шрёдингера и векторного уравнения длиннокороткого резонанса. Для уиземовских уравнений описание локальных мультигамильтоновых структур было дано в работах $[1,15]$ алгебро-геометрическим методом. В данной работе построены третьи гамильтоновы структуры для всех указанных систем общим дифференциально-геометрическим методом. При этом оказывается, что для выяснения вопроса о наличии второй и третьей гамильтоновых структур и их типе (локальная, нелокальная с метрикой постоянной кривизны, общая нелокальная) достаточно знать степени однородности аннуляторов первой гамильтоновой структуры, которые, как правило, известны заранее. Мы доказываем также простой критерий егоровости метрики (теорема 1). Теоремы о тригамильтоновых структурах в общем случае формулируются и доказываются в $\$ \S 2-4$ и применяются к упомянутым выше примерам в следующих параграфах.

Кроме приложений к интегрируемым диагонализируемым системам гидродинамического типа рассматриваемая нами теория имеет известные приложения в теории фробениусовых многообразий и конформной топологической теории поля для решения уравнений ассоциативности Виттена-Дийкграафа-Э. ВерлиндеГ. Верлинде [18].

*Исследования первого автора получили частичную финансовую поддержку РФФИ (гранты 00-01-00210 и 00-01-00366), а второго автора - частичную финансовую поддержку INTAS (грант 99-01782) и Минобразования РФ (грант Е00-1.0-57). 
Кратко сформулируем необходимые для дальнейшего результаты (см. [13]).

Для исходной диагонализируемой системы гидродинамического типа (1) существуют инварианты Римана $r^{i}=r^{i}(u), i=1, \ldots, N$, т. е. переменные, в которых матрица скоростей $\left(v_{k}^{i}\right)$ системы $(1)$ имеет диагональную форму (суммирования по повторяющимся индексам нет, если не оговорено противное):

$$
r_{t}^{i}=v^{i}(r) r_{x}^{i}, \quad i=1, \ldots, N
$$

В дальнейшем мы предполагаем, что $v^{i} \neq v^{k}$ при $i \neq k$ и система (2) нерасnaдающцаяся: $\partial_{i} v^{k} \neq 0, i \neq k$. Исходя из $v^{i}(r)$, найдем коэффициенты Ламе $H_{i}(u)$ как некоторые решения переопределенной системы

$$
\partial_{k} \ln H_{i}=\Gamma_{i k}^{i}=\frac{\partial_{k} v^{i}}{v^{k}-v^{i}}, \quad i \neq k ;
$$

здесь $\partial_{k} \equiv \partial / \partial r^{k}$, а $\Gamma_{i k}^{i}-$ символ Кристоффеля связности Леви-Чевита, соответствующей метрике, входящей в гамильтонов оператор $\widehat{A}^{i j}$ данной системы (см. (8), (9) ниже); также найдем коэффициенты вращения (см. $[13,17])$

$$
\beta_{i k}=\frac{\partial_{i} H_{k}}{H_{i}}, \quad i \neq k ;
$$

условиями совместности систем (3) или (4) являются условия полугамильтоновости $[12,13]$

$$
\partial_{i} \beta_{j k}=\beta_{j i} \beta_{i k} \Longleftrightarrow \partial_{j} \frac{\partial_{k} v^{i}}{v^{k}-v^{i}}=\partial_{k} \frac{\partial_{j} v^{i}}{v^{j}-v^{i}}, \quad i \neq j \neq k .
$$

При этом диагональные коэффициенты системы (2) можно представить в виде $v^{i}(r)=\bar{H}_{i} / H_{i}$, где $H_{i}-$ коэффициенты Ламе, а $\bar{H}_{i}-$ некоторые решения системы

$$
\partial_{i} \bar{H}_{k}=\beta_{i k} \bar{H}_{i}, \quad i \neq k .
$$

Локальная гамильтонова структура типа Дубровина-Новикова для полугамильтоновой системы (2) существует, если и только если

$$
\partial_{i} \beta_{i k}+\partial_{k} \beta_{k i}+\sum_{m \neq i, k} \beta_{m i} \beta_{m k}=0, \quad i \neq k .
$$

В этом случае система гидродинамического типа в виде (1) или (2) записывается в гамильтоновой форме

$$
u_{t}^{i}=\left\{u^{i}, H\right\}=\sum_{j=1}^{N} \widehat{A}^{i j} \frac{\delta H}{\delta u^{j}}=\sum_{k=1}^{N}\left(\nabla^{i} \nabla_{k} h\right) u_{x}^{k}
$$

с гамильтоновым оператором

$$
\widehat{A}^{i j}=g^{i j}(u) \frac{d}{d x}-\sum_{s, k} g^{i s}(u) \Gamma_{s k}^{j}(u) u_{x}^{k},
$$

где $g^{i j}=g^{j i}$ - невырожденная метрика нулевой кривизны, а связность $\Gamma_{s k}^{j}$ симметрична и согласована с метрикой (здесь $H=\int h(u) d x-$ гамильтониан гидродинамического типа). В инвариантах Римана метрика диагональна, $g_{i i}=H_{i}^{2}$ $\left(g^{i i} g_{i i}=1\right)$, а обращение в нуль тензора Римана эквивалентно (7). Тем самым с 
каждой локальной гамильтоновой структурой системы (2) связана система ортогональных криволинейных координат $r^{i}$ в плоском $N$-мерном псевдоевклидовом пространстве. Отметим, что в случае псевдоримановой диагональной метрики (а именно такие и встречаются в приложениях - этот факт поясняет теорема 7 ниже) некоторые из величин $H_{i}, \beta_{i k}$ являются чисто мнимыми.

Каждый коэффициент $H_{i}$ определяется из $(3)$ с точностью до умножения на функцию одного переменного: $H_{i}=\mu_{i}^{-1 / 2}\left(r^{i}\right) H_{i}^{\text {(fix) }}$. Условие $(7)$ при этом принимает вид

$$
\frac{1}{2} \mu_{i}^{\prime} \beta_{i k}^{(\mathrm{fix})}+\frac{1}{2} \mu_{k}^{\prime} \beta_{k i}^{(\mathrm{fix})}+\mu_{i} \partial_{i} \beta_{i k}^{(\mathrm{fix})}+\mu_{k} \partial_{k} \beta_{k i}^{(\mathrm{fix})}+\sum_{m \neq i, k} \mu_{m} \beta_{m i}^{(\mathrm{fix})} \beta_{m k}^{(\mathrm{fix})}=0 .
$$

В силу линейности системы уравнений (10) относительно $\mu_{i}$ легко заметить, что любые две гамильтоновы структуры (9), связанные с данной диагональной системой гидродинамического типа с невырожденными метриками $g_{i j}$, автоматически совместны. Как было показано в работе [20], система уравнений (10) на $\mu_{i}\left(r^{i}\right)$ не может иметь больше, чем $N+1$, линейно независимых решений; в общем случае для заданной полугамильтоновой системы она решений вообще не имеет.

Система гидродинамического типа (1) может также иметь нелокальную гамильтонову структуру (8) с нелокальным гамильтоновым оператором [11]

$$
\widehat{A}^{i j}=g^{i j} d / d x-\sum_{s, k} g^{i s} \Gamma_{s k}^{j} u_{x}^{k}+\sum_{\alpha, \beta, n, m} \varepsilon_{\alpha \beta} w_{n}^{i,(\alpha)} u_{x}^{n}(d / d x)^{-1} w_{m}^{j,(\beta)} u_{x}^{m}
$$

здесь $w_{n}^{i,(\alpha)}(u)$ - матричные коэффициенты коммутирующих с (1) потоков гидродинамического типа

$$
u_{t_{(\alpha)}}^{i}=\sum_{j=1}^{N} w_{j}^{i,(\alpha)}(u) u_{x}^{j}
$$

в инвариантах Римана $w_{n}^{i,(\alpha)}$ диагональны, $w_{i}^{i,(\alpha)}=H_{i}^{(\alpha)} / H_{i}$, где $H_{i}^{(\alpha)}-$ набор решений системы (6), такой, что

$$
\partial_{i} \beta_{i k}+\partial_{k} \beta_{k i}+\sum_{m \neq i, k} \beta_{m i} \beta_{m k}=\sum_{\alpha, \beta} \varepsilon_{\alpha \beta} H_{i}^{(\alpha)} H_{k}^{(\beta)}, \quad i \neq k
$$

$\varepsilon_{\alpha \beta}=\varepsilon_{\beta \alpha}=$ const. Таким образом, метрика $g^{i j}(u)$ в указанном нелокальном гамильтоновом операторе не является плоской; в случае, когда в (13) имеется одно слагаемое $H_{i} H_{k}$, образованное из коэффициентов Ламе данной метрики $H_{i}=\sqrt{g_{i i}}$, получаем метрику постоянной кривизны [5].

\section{§2. Егоровские системы гидродинамического типа}

Как показано в $[12,13]$, диагональная полугамильтонова система $(2)$ имеет бесконечный набор коммутирующих потоков гидродинамического типа

$$
r_{y}^{i}=w^{i}(r) r_{x}^{i}, \quad i=1, \ldots, N,
$$

коэффициенты $w^{i}(r)$ которых находятся как решения совместной переопределенной системы линейных уравнений в частных производных первого порядка

$$
\partial_{k} w^{i}=\Gamma_{i k}^{i}\left(w^{k}-w^{i}\right), \quad i \neq k .
$$


Система (2) также имеет бесконечный набор гидродинамических законов сохранения $d p(r) / d t=d q(r) / d x$, где плотности законов сохранения $p(r)$ определяются из совместной системы

$$
\partial_{i} \partial_{k} p=\Gamma_{i k}^{i} \partial_{i} p+\Gamma_{i k}^{k} \partial_{k} p, \quad i \neq k .
$$

Система (15) с помощью подстановки $w^{i}=\widetilde{H}_{i} / H_{i}$ может быть переписана в виде (6), а система (16) после подстановки $\partial_{i} p=\psi_{i} H_{i}-$ как система

$$
\partial_{k} \psi_{i}=\beta_{i k} \psi_{k}, \quad i \neq k,
$$

сопряженная к системе (6).

ОПРЕДЕЛЕНИЕ 1. Ортогональная система координат, связанная с диагональной метрикой $g_{i i}=H_{i}^{2}$, называется егоровской, если

$$
\beta_{i k}=\beta_{k i} \text {. }
$$

При этом метрика будет потенцииальной: $g_{i i}=\partial_{i} a(r)$ для некоторой функции $a(r)$.

Соответствующие системы гидродинамического типа будем также называть егоровскими. Условие (18) не инвариантно относительно естественной замены инвариантов Римана $r^{i} \rightarrow \varphi^{i}\left(r^{i}\right)$ или замены $H_{i} \rightarrow \mu_{i}^{-1 / 2}\left(r^{i}\right) H_{i}$; инвариантным условием для нераспадающейся системы $\left(\beta_{i k} \neq 0\right)$ при $N \geqslant 3$ (см. [3, 17]) является равенство

$$
\beta_{i k} \beta_{k j} \beta_{j i}=\beta_{i j} \beta_{j k} \beta_{k i}, \quad i \neq j \neq k .
$$

Если система гидродинамического типа является егоровской, то $\partial_{i} p=H_{i} \bar{H}_{i}$ и системы (6) и (17) совпадают. Поскольку интегрируемые системы гидродинамического типа имеют бесконечный набор независимых законов сохранения $\partial_{t} p_{k}(u)=\partial_{x} q_{k}(u), k=1,2, \ldots$, они могут быть переписаны в консервативной форме

$$
\partial_{t} a^{\alpha}=\partial_{x} q^{\alpha}(a), \quad \alpha=1, \ldots, N,
$$

где $a^{\alpha}=a^{\alpha}(u)$ - плотности законов сохранения, а $q^{\alpha}(a)-$ соответствующие токи.

Докажем следующий критерий егоровости системы [8]:

Теорема 1. Диагонализируемая нераспадающаяся полугамильтонова система (1) имеет пару законов сохранения вида

$$
a_{t}=b_{x}, \quad b_{t}=c_{x},
$$

если и только если она является егоровской. В этом случае в инвариантах Puмara $\partial_{i} a=H_{i}^{2}, \partial_{i} b=H_{i} \bar{H}_{i}, \partial_{i} c=\bar{H}_{i}^{2}$, где $\bar{H}_{i}=v^{i}(r) H_{i}$.

ДокАЗАТЕЛьство. Условие (21) может быть переписано так:

$$
\begin{aligned}
\partial_{i} a & =\psi_{i} H_{i}, & \partial_{i} b & =\psi_{i} \bar{H}_{i}, \\
\partial_{i} b & =\bar{\psi}_{i} H_{i}, & \partial_{i} c & =\bar{\psi}_{i} \bar{H}_{i},
\end{aligned}
$$

где $\psi_{i}, \overline{\psi_{i}}$ - решения системы $(17)$, откуда $\bar{\psi}_{i} H_{i}=\psi_{i} \bar{H}_{i}$. Продифференцировав $\bar{\psi}_{i}=\psi_{i} \bar{H}_{i} / H_{i}$ по инварианту Римана $r^{k}(i \neq k)$ и разделив на $v_{i}-v_{k}=\bar{H}_{i} / H_{i}-$ $\bar{H}_{k} / H_{k}$, из (17) получим

$$
\frac{\psi_{k}}{H_{k}} \beta_{i k}=\frac{\psi_{i}}{H_{i}} \beta_{k i},
$$


что влечет за собой (19) для случая, когда число уравнений $N$ больше 2. Отметим, что условие нераспадения системы $\left(\partial_{i} v^{k} \neq 0, i \neq k\right)$ означает в силу $(3),(4)$, что $\beta_{k i} \neq 0$ при $i \neq k$, и гарантирует в силу $(24)$, что $\psi_{i} \neq 0, \partial_{i} a \neq 0$ для любого $i$. Для доказательства егоровости в случае, если система (1) содержит только два уравнения, положим $q_{i}=\psi_{i} / H_{i}=\bar{\psi}_{i} / \bar{H}_{i}$. Из (23) получаем $\partial_{i}\left(q_{k} H_{k}^{2}\right)=\partial_{k}\left(q_{i} H_{i}^{2}\right)$ и $\partial_{i}\left(q_{k} \bar{H}_{k} H_{k}\right)=\partial_{k}\left(q_{i} \bar{H}_{i} H_{i}\right)$, откуда, используя $(24)$, приходим к равенствам $H_{i}^{2} \partial_{k} q_{i}=H_{k}^{2} \partial_{i} q_{k}$ и $\bar{H}_{i} H_{i} \partial_{k} q_{i}=\bar{H}_{k} H_{k} \partial_{i} q_{k}$. Деля полученные выражения друг на друга (в предположении, что $\partial_{k} q_{i} \neq 0, i \neq k$ ), имеем $v_{i}=v_{k}$, что противоречит предположению $v_{i} \neq v_{k}$ при $i \neq k$; значит, $\partial_{k} q_{i}=0$. Но поскольку инварианты Римана и определены с точностью до замены $r^{i} \rightarrow \varphi^{i}\left(r^{i}\right)$, функции $q_{i}\left(r^{i}\right)=\psi_{i} / H_{i}$ в $(24)$ всегда можно положить тождественно равными единице, т. е. метрика потенциальная и $\partial_{i} a=H_{i}^{2}, \partial_{i} b=H_{i} \bar{H}_{i}, \partial_{i} c=\bar{H}_{i}^{2}$.

Обратно, если метрика потенциальна, $H_{i}^{2}=\partial_{i} a$, то выполнены условия (18). Имеем $a_{t}=\partial_{i} a \cdot r_{t}^{i}=H_{i}^{2} r_{t}^{i}=H_{i}^{2} v^{i}(r) r_{x}^{i}=H_{i} \bar{H}_{i} r_{x}^{i}=b_{x}, b_{t}=\partial_{i} b \cdot r_{t}^{i}=H_{i} \bar{H}_{i} r_{t}^{i}=$ $H_{i} \bar{H}_{i} v^{i}(r) r_{x}^{i}=\bar{H}_{i}^{2} r_{x}^{i}=c_{x}$.

Отметим, что в доказанной теореме условие плоскости метрики не было нами использовано. Самим Д. Ф. Егоровым изучались потенциальные метрики с дополнительным условием нулевой кривизны. Мы в этой работе будем называть егоровскими потенциальные метрики произвольной кривизны. Так как все коммутирующие потоки имеют одну и ту же метрику (см. (3), (15)), то мы получаем следующий результат.

СлЕДСТВИЕ 1. Произвольный коммутирующий поток (14) егоровской полугамильтоновой системь гидродинамического типа имеет пару законов сохранения вида (21) $\partial_{y} a=\partial_{x} h, \partial_{y} h=\partial_{x} g$.

Очевидно, что если система гидродинамического типа (2) имеет локальную гамильтонову структуру (8), то существует связь $w_{k}^{i}(u)=\nabla^{i} \nabla_{k} p$ между решениями уравнений на плотности законов сохранения (16) и решениями уравнений на коммутирующие потоки (15). Раскрывая операцию ковариантного дифференцирования $\nabla^{i}$ и подставляя (в инвариантах Римана) выражения $w^{i}(r)=\bar{H}_{i} / H_{i}$ и $\partial_{i} p=\psi_{i} H_{i}$, получим связь между решениями прямой и сопряженной задач $(6)$ и $(17)$ :

$$
\bar{H}_{i}=\partial_{i} \psi_{i}+\sum_{m \neq i} \beta_{m i} \psi_{m}
$$

Если система гидродинамического типа имеет вторую локальную гамильтонову структуру, то, как уже отмечалось, диагональные коэффициенты ее метрики $g_{(2)}^{i i}$ могут отличаться лишь множителями, зависящими от соответствующих инвариантов Римана $g_{(2)}^{i i}=\mu_{i}\left(r^{i}\right) g_{(1)}^{i i}$. Произведем замену инвариантов Римана $r^{i} \rightarrow \varphi^{i}\left(r^{i}\right)$ так, что $\mu_{i}\left(r^{i}\right) \rightarrow r^{i}$ (в предположении, что $\left.\mu_{i}^{\prime}\left(r^{i}\right) \neq 0\right)$, т.е. $g_{(2)}^{i i}=r^{i} g_{(1)}^{i i}$. Для связности, порожденной второй плоской метрикой $g_{(2)}^{i i}$, аналогично (25) получим

$$
\widetilde{H}_{i}=\frac{1}{2} \psi_{i}+r^{i} \partial_{i} \psi_{i}+\sum_{m \neq i} r^{m} \beta_{m i} \psi_{m}
$$


Если метрика $g_{(1)}^{i i}$ егоровская, то из формул $(25)$ и (26) следует, что

$$
\bar{H}_{i}=\delta \psi_{i}, \quad \widetilde{H}_{i}=(\widehat{R}+1 / 2) \psi_{i},
$$

где $\delta=\sum \partial_{k}$ - оператор сдвига и $\widehat{R}=\sum r^{k} \partial_{k}$ - оператор растяжения (см. [10]). Суммируем сказанное выше: при подходящем выборе инвариантов Римана, если егоровская система гидродинамического типа имеет одну локальную гамильтонову структуру, то $\delta \beta_{i k}=0$, т. е. коэффициенты вращения ортогональных систем координат зависят только от разностей инвариантов Римана, $\beta_{i k}=$ $\beta_{i k}\left(r^{m}-r^{n}\right)$; если же егоровская система гидродинамического типа еще имеет и вторую локальную гамильтонову структуру с метрикой $g_{(2)}^{i i}=r^{i} g_{(1)}^{i i}$, то из $(10),(5)$ получаем эквивалентное условие однородности $\widehat{R} \beta_{i k}=-\beta_{i k}$, т. е. коэффициенты вращения ортогональных систем координат являются однородными функциями степени однородности -1. Как показано в [13], условие егоровости метрики для диагонализируемой гамильтоновой системы фактически служит выражением ее инвариантности относительно группы преобразований Галилея $(x, t) \rightarrow(x-V \cdot t, t)$. Однородность коэффициентов вращения в известных примерах соответствует естественному физическому условию инвариантности относительно выбора единиц измерения, что приводит к однородности $v_{i}(r)$. Таким образом, наличие двух локальных гамильтоновых структур для рассматриваемых нами физических примеров систем гидродинамического типа служит проявлением простейших фундаментальных условий инвариантности. Как уже отмечалось выше, соответствующие гамильтоновы структуры автоматически оказываются совместными.

В дальнейшем мы для краткости будем говорить «однородные коэффициенты вращения», подразумевая, что степень их однородности равна всегда -1 : $\widehat{R} \beta_{i k}=-\beta_{i k}$. Соответствующие криволинейные ортогональные системы координат и диагональные системы гидродинамического типа (2) также будем просто называть однородными.

\section{§3. Аннуляторы первой локальной гамильтоновой структуры и сигнатура ее метрики для однородных егоровских систем}

Аннуляторами локальной гамильтоновой структуры называются плотности законов сохранения $a^{\alpha}(u)$, которые определяются условием обращения в нуль коммутирующих потоков,

$$
0=\nabla^{i} \nabla_{k} a^{\alpha}, \quad \alpha=1, \ldots, N .
$$

Переопределенная система (28) имеет $N$-мерное пространство решений, которое задает плоские координаты для метрики $g_{i i}=H_{i}^{2}$ гамильтоновой структуры. Введя $\psi_{i}^{(\alpha)}=\partial_{i} a^{\alpha} / H_{i}$ в инвариантах Римана, перепишем $(28)$ в виде

$$
\partial_{k} \psi_{i}^{(\alpha)}=\beta_{i k} \psi_{k}^{(\alpha)}, \quad 0=\partial_{i} \psi_{i}^{(\alpha)}+\sum_{m \neq i} \beta_{m i} \psi_{m}^{(\alpha)}, \quad \alpha=1, \ldots, N \text {. }
$$

Следующие две теоремы были доказаны в [14] и позднее изложены в [18].

Теорема 2. Однороднье решения $\psi_{i}^{(\alpha)}$ системы (29) для егоровской ортогональной системь координат, коэффициенты вращения которой однороднь и зависят только от разности инвариантов Римана, являются собственными 
векторами кососимметрической матрицьь $\boldsymbol{B}$ с элементами $B_{i j}=\left(r^{i}-r^{j}\right) \beta_{i j}$, имеющей постоянные собственные числа.

ДоказАТЕЛьство. Имеем

$$
\begin{aligned}
\widehat{R} \psi_{i}^{(\alpha)} & =r^{i} \psi_{i, i}^{(\alpha)}+\sum_{m \neq i} r^{m} \partial_{m} \psi_{i}^{(\alpha)} \\
& =\sum_{m \neq i} r^{m} \beta_{m i} \psi_{m}^{(\alpha)}-r^{i} \sum_{m \neq i} \beta_{m i} \psi_{m}^{(\alpha)}=\sum_{m \neq i}\left(r^{m}-r^{i}\right) \beta_{m i} \psi_{m}^{(\alpha)}=c_{\alpha} \psi_{i}^{(\alpha)} .
\end{aligned}
$$

Постоянство собственных чисел $c_{\alpha}(r)$ матрицы $\boldsymbol{B}$ доказывается следующим образом: $\partial_{i}\left(\widehat{R} \psi_{k}^{(\alpha)}\right)=\widehat{R}\left(\partial_{i} \psi_{k}^{(\alpha)}\right)+\partial_{i} \psi_{k}^{(\alpha)}=\widehat{R}\left(\beta_{k i} \psi_{i}^{(\alpha)}\right)+\beta_{k i} \psi_{i}^{(\alpha)}=\widehat{R}\left(\beta_{k i}\right) \psi_{i}^{(\alpha)}+$ $\beta_{k i} \widehat{R}\left(\psi_{i}^{(\alpha)}\right)+\beta_{k i} \psi_{i}^{(\alpha)}=-\beta_{k i} \psi_{i}^{(\alpha)}+c_{\alpha} \psi_{i}^{(\alpha)} \beta_{k i}+\beta_{k i} \psi_{i}^{(\alpha)}=c_{\alpha} \psi_{i}^{(\alpha)} \beta_{k i}$, т. е. $\partial_{i}\left(c_{\alpha} \psi_{k}^{(\alpha)}\right)$ $=\partial_{i}\left(c_{\alpha}\right) \psi_{k}^{(\alpha)}+c_{\alpha} \beta_{k i} \psi_{i}^{(\alpha)}=c_{\alpha} \psi_{i}^{(\alpha)} \beta_{k i}$, откуда $\partial_{i}\left(c_{\alpha}\right) \equiv 0$.

Величины $\psi_{i}^{(\alpha)}$ также будем называть аннуляторами.

Поскольку для рассматриваемого нами случая однородных вещественных гиперболических систем (2) степени однородности любых плотностей законов сохранения, в том числе аннуляторов, - вещественные числа, собственные значения кососимметрической матрицы $\boldsymbol{B}$ также вещественны. Данный факт объясняется тем, что для указанного класса систем гидродинамического типа метрика обязана быть псевдоевклидовой (см. теорему 7 ниже), т. е. некоторые коэффициенты Ламе $H_{i}=\sqrt{g_{i i}}$ и коэффициенты вращения $\beta_{i k}$ будут чисто мнимыми. Матрица $\boldsymbol{B}$, таким образом, имеет как вещественные, так и чисто мнимые элементы; для таких матриц уже нельзя утверждать, что имеется полный набор собственных векторов: например, при $N=3$ для егоровской однородной метрики сигнатуры 1 можно выбрать коэффициенты $\beta_{i j}$ так, что $\left(r^{1}-r^{2}\right)^{2} \beta_{12}^{2}+$ $\left(r^{1}-r^{3}\right)^{2} \beta_{13}^{2}+\left(r^{2}-r^{3}\right)^{2} \beta_{23}^{2}=0,-$ данное условие, как легко проверить, совместимо с приведенными ниже уравнениями (30) на $\beta_{i k}$. В таком случае матрица $\boldsymbol{B}$ будет иметь один собственный вектор и два корневых с собственным значением 0. Отметим, что множество егоровских ортогональных систем координат заданной размерности, коэффициенты вращения которых являются однородными и зависят только от разностей инвариантов Римана, конечнопараметрическое, и их описание сводится к описанию решений совместной пфаффовой системы

$$
\partial_{i} \beta_{j k}=\beta_{j i} \beta_{i k}, \quad \delta \beta_{i k}=0, \quad \widehat{R} \beta_{i k}=-\beta_{i k}, \quad i \neq j \neq k ;
$$

ее решение определяется заданием произвольной симметричной матрицы $\left(\beta_{i k}\right)$ (где $\beta_{i i}$ не определены) в начальной точке $\left(r_{(0)}^{i}\right)$.

Однако все известные нам физические примеры диагонализируемых однородных егоровских систем, а также возникающие в конформной топологической теории поля егоровские ортогональные системы координат, коэффициенты вращения которых являются однородными и зависят только от разностей инвариантов Римана, приводят к матрицам $\boldsymbol{B}$, имеющим полный набор собственных векторов, причем, как правило, для физических систем нам заранее известны степени однородности аннуляторов первой локальной скобки Пуассона. Поэтому мы всюду в дальнейшем предполагаем, что выполнено условие полупростоть - наличия полного набора собственных векторов матрицы $\boldsymbol{B}$ в некоторой точке $\left(r_{(0)}^{i}\right)$. Как показано в [18], описание полупростых решений системы (30) сводится к решению уравнения Пенлеве VI. 
Теорема 3. Для полупростой матрицьь $\boldsymbol{B}$ все аннуляторы $\psi_{i}^{(\alpha)}$, соответствующие плоской однородной егоровской системе координат, могут быть выљраны однородными функииями:

$$
\boldsymbol{B} \vec{\psi}^{(\alpha)}=\widehat{R} \vec{\psi}^{(\alpha)}=c_{\alpha} \vec{\psi}^{(\alpha)}, \quad \alpha=1, \ldots, N,
$$

где $c_{\alpha}$ (степени однородности аннуляторов) одновременно являются собственными числами матрицьь $\boldsymbol{B}$.

ДокАЗАТЕЛьство. В случае симметричной матрицы $\left(\beta_{i k}\right)$, т. е. для егоровских метрик, второе из соотношений (29) превращается в $\delta \psi_{i}^{(\alpha)}=\partial_{i} \psi_{i}^{(\alpha)}+$ $\sum_{m \neq i} \beta_{i m} \psi_{m}^{(\alpha)}=\partial_{i} \psi_{i}^{(\alpha)}+\sum_{m \neq i} \partial_{m} \psi_{i}^{(\alpha)}=0$. Таким образом, $\psi_{i}^{(\alpha)} \quad$ (возможно, неоднородные) удовлетворяют системе

$$
\delta \psi_{i}^{(\alpha)}=0, \quad \partial_{i} \psi_{k}^{(\alpha)}=\beta_{i k} \psi_{i}^{(\alpha)}, \quad i \neq k .
$$

Данная система, как легко проверить, совместна, начальные данные для нее задаются $N$ константами - значениями функции $\vec{\psi}^{(\alpha)}$ в некоторой точке $\left(r_{(0)}^{i}\right)$. Повторяя вычисления, проделанные в доказательстве предыдущей теоремы, получаем, что для любого решения $\bar{\psi}_{i}$ и любого $c=$ const имеют место равенства $\partial_{i}\left(\widehat{R} \bar{\psi}_{k}-c \bar{\psi}_{k}\right)=\beta_{i k}\left(\widehat{R}_{i}-c \bar{\psi}_{i}\right), \delta\left(\widehat{R} \bar{\psi}_{k}-c \bar{\psi}_{k}\right)=0$, т. е. $\widetilde{\psi}_{i}=\widehat{R} \bar{\psi}_{i}-c \bar{\psi}_{i}$ также удовлетворяет системе (32). Следовательно, выбрав для системы (32) начальные данные $\vec{\psi}^{(\alpha)}\left(r_{(0)}^{i}\right)$ - базис собственных векторов матрицы $\boldsymbol{B}$ в этой точке $\left(r_{(0)}^{i}\right)$, получим, что $\widetilde{\psi}_{i}^{(\alpha)}=\widehat{R} \bar{\psi}_{i}^{(\alpha)}-c_{\alpha} \bar{\psi}_{i}^{(\alpha)}$ также будут решениями системы $(32)$, равными 0 в точке $\left(r_{(0)}^{i}\right)$, т. е. $\widetilde{\psi}_{i}^{(\alpha)} \equiv 0$, что и требовалось доказать.

ОПРЕДЕЛЕНИЕ 2. Плотностью импульса $P$ системы гидродинамического типа с локальной гамильтоновой структурой называется квадратичное выражение

$$
P=\frac{1}{2} \sum_{\alpha, \beta} g_{\alpha \beta} a^{\alpha} a^{\beta},
$$

где невырожденная матрица $g_{\alpha \beta}-$ (постоянная) метрика системы $(2)$ в плоских координатах-аннуляторах $a^{\alpha}$.

Очевидно (см. [2]), что в координатах-аннуляторах $a^{\alpha}$ система гидродинамического типа (1) имеет вид

$$
a_{t}^{\alpha}=\frac{d}{d x}\left(\sum_{\beta=1}^{N} g^{\alpha \beta} \frac{\partial h}{\partial a^{\beta}}\right),
$$

где $h(u)$ - плотность гамильтониана. Плотность импульса дает интеграл любой гамильтоновой системы гидродинамического типа $(34)$ с данной метрикой $g^{\alpha \beta}$ и порождает тривиальный коммутирующий поток $u_{t}^{i}=u_{x}^{i}$.

Отметим здесь следующее утверждение (независимо доказанное в нескольких работах разных авторов):

Теорема 4. Если система гидродинамического типа (1) (не обязательно диагонализируемая) имеет $N+1$ линейно независимых гидродинамических законов сохранения и одна из их плотностей квадратично выражается через остальные (см. (33)), то такая система имеет локальную гамильтонову структуру (34) с постоянной метрикой $g_{\alpha \beta}$. 
ДокАЗАТЕЛЬСтво. Пусть $\partial_{t} a^{\alpha}=\partial_{x} q^{\alpha}, \partial_{t} P=\partial_{t}\left[\frac{1}{2} \sum_{\alpha, \beta} g_{\alpha \beta} a^{\alpha} a^{\beta}\right]=\partial_{x} Q(a)$. Отсюда вытекает, что $d Q=\sum_{\alpha, \beta} g_{\alpha \beta} a^{\alpha} d q^{\beta}(a)=\sum_{\alpha, \beta} d\left[g_{\alpha \beta} a^{\alpha} q^{\beta}\right]-\sum_{\alpha, \beta} g_{\alpha \beta} q^{\beta} d a^{\alpha}$. Следовательно, $\sum_{\beta} g_{\alpha \beta} q^{\beta} \equiv \partial h / \partial a^{\alpha}$ для некоторой функции $h(u)$, а значит, система (20) принимает форму (34).

В работе [7] сформулирована, а в [25] доказана теорема, обобщающая предыдущую на случай метрики постоянной кривизны:

Теорема 5. Пусть система гидродинамического типа, записанная в форме законов сохранения $\partial_{t} c^{\alpha}=\partial_{x} b^{\alpha}$, имеет дополнительную плотность закона сохранения $p$, связанную квадратичным соотношением с плотностями $c^{\alpha}$ :

$$
p-\frac{\varepsilon}{2} p^{2}=\frac{1}{2} \sum_{\alpha, \beta} \bar{g}_{\alpha \beta} c^{\alpha} c^{\beta},
$$

где $\bar{g}_{\alpha \beta}$ - постоянная невырожденная матрица. Тогда данная система в полевых переменных $c^{\alpha}$ имеет нелокальную гамильтонову структуру

$$
c_{t}^{\alpha}=\partial_{x}\left[\sum_{\beta=1}^{N}\left(\bar{g}^{\alpha \beta}-\varepsilon c^{\alpha} c^{\beta}\right) \frac{\partial h}{\partial c^{\beta}}+\varepsilon c^{\alpha} h\right],
$$

ассоциированную с метрикой $g^{\alpha \beta}=\bar{g}^{\alpha \beta}-\varepsilon c^{\alpha} c^{\beta}$ постоянной кривизнь $\varepsilon$, где $\left(\bar{g}^{\alpha \beta}\right)$ - обратная матрица $\kappa\left(\bar{g}_{\alpha \beta}\right)$.

ДоКАЗАТЕЛЬСтво. Из формул $\partial_{t} c^{\alpha}=\partial_{x} b^{\alpha}, p_{t}=\partial_{x} Q(c)$ и (35) получаем

$$
d Q=\sum_{\alpha, \beta} \bar{g}_{\alpha \beta} \frac{c^{\beta}}{1-\varepsilon p} d b^{\alpha} .
$$

Полагая $q^{\alpha}=c^{\alpha} /(1-\varepsilon p)$, получим, что $\sum_{\beta} \bar{g}_{\alpha \beta} b^{\beta}=\partial s / \partial q^{\alpha}$ с некоторым потенциалом $s$. Отсюда видно, что

$$
p_{t}=\partial_{x}\left[\sum_{\beta} q^{\beta} \frac{\partial s}{\partial q^{\beta}}-s\right] \quad \text { и } \quad c_{t}^{\alpha}=\partial_{x}\left[\sum_{\beta} \bar{g}^{\alpha \beta} \frac{\partial s}{\partial q^{\beta}}\right] .
$$

Так как

$$
\frac{\partial s}{\partial q^{\alpha}}=(1-\varepsilon p)\left[\frac{\partial s}{\partial c^{\alpha}}-\sum_{\beta, \gamma} \varepsilon c^{\gamma} \frac{\partial s}{\partial c^{\gamma}} \bar{g}_{\alpha \beta} c^{\beta}\right],
$$

то немедленно получаем $(36)$ с $h=(1-\varepsilon p) s$.

Разумеется, соотношение (35) можно сдвигом $p \rightarrow p+$ const превратить в чисто квадратичное соотношение; однако данная в (35) форма проясняет ситуацию с предельным переходом $\varepsilon \rightarrow 0$.

Теорема 6. Имеют место следующие соотношения для компонент любой (не обязательно егоровской или однородной) плоской метрики в плоских координатах-аннуляторах:

$$
\sum_{\alpha, \beta} g_{\alpha \beta} \psi_{i}^{(\alpha)} \psi_{k}^{(\beta)}=\delta_{i k}, \quad g^{\alpha \beta}=\sum_{i=1}^{N} \psi_{i}^{(\alpha)} \psi_{i}^{(\beta)} .
$$


В частном случае егоровской метрики этот результат был получен в [19]. ДокАЗАТЕЛЬСтво. Для Плотности импульса $P$ (см. (33)) имеем

$$
\partial_{i} P=\Psi_{i} H_{i}=\sum_{\alpha, \beta} g_{\alpha \beta} a^{\beta} \psi_{i}^{(\alpha)} H_{i} \Longrightarrow \Psi_{i}=\sum_{\alpha, \beta} g_{\alpha \beta} a^{\beta} \psi_{i}^{(\alpha)} .
$$

Дифференцируя по $r^{k}$ и используя (17), получаем $\left.\sum_{\alpha, \beta} g_{\alpha \beta} \psi_{i}^{(\alpha)} \psi_{k}^{(\beta)}\right|_{i \neq k}=0$. Принимая во внимание, что $P$ порождает поток $u_{t}^{i}=u_{x}^{i}$, получаем, дифференцируя по $r^{i}$, равенство $\sum_{\alpha, \beta} g_{\alpha \beta} \psi_{i}^{(\alpha)} \psi_{i}^{(\beta)}=1$, что и дает первую из формул (37). Домножая ее на $\psi_{k}^{(\gamma)}$ и суммируя по $k$, приходим к соотношению

$$
\sum_{k=1}^{N}\left(\sum_{\alpha, \beta} g_{\alpha \beta} \psi_{i}^{(\alpha)} \psi_{k}^{(\beta)}\right) \psi_{k}^{(\gamma)}=\sum_{\alpha=1}^{N} \psi_{i}^{(\alpha)}\left(\sum_{\beta=1}^{N} g_{\alpha \beta} \sum_{k=1}^{N} \psi_{k}^{(\beta)} \psi_{k}^{(\gamma)}\right)=\psi_{i}^{(\gamma)} .
$$

В силу линейной независимости векторов $\vec{\psi}^{(\alpha)}$ отсюда вытекает равенство

$$
\sum_{\beta=1}^{N} g_{\alpha \beta}\left(\sum_{k=1}^{N} \psi_{k}^{(\beta)} \psi_{k}^{(\gamma)}\right)=\delta_{\alpha}^{\gamma}
$$

т. е. вторая формула (37).

Вернемся к случаю егоровской ортогональной системы координат, коэффициенты вращения которой являются однородными и зависят только от разностей инвариантов Римана. Тогда

$$
\begin{aligned}
\widehat{R} g^{\alpha \beta}=0 & =\widehat{R} \sum_{i=1}^{N} \psi_{i}^{(\alpha)} \psi_{i}^{(\beta)}=\sum_{i=1}^{N} \psi_{i}^{(\alpha)}\left(\widehat{R} \psi_{i}^{(\beta)}\right)+\sum_{i=1}^{N} \psi_{i}^{(\beta)}\left(\widehat{R} \psi_{i}^{(\alpha)}\right) \\
& =\left(c_{\alpha}+c_{\beta}\right) \sum_{i=1}^{N} \psi_{i}^{(\alpha)} \psi_{i}^{(\beta)} .
\end{aligned}
$$

Отсюда следует, что либо $c_{\alpha}+c_{\beta} \neq 0$ и тогда соответствующая компонента матрицы $\left(g^{\alpha \beta}\right)$ равна 0 , либо $c_{\alpha}+c_{\beta}=0$ и тогда соответствующая компонента матрицы $\left(g^{\alpha \beta}\right)$ отлична от 0 (некоторые из них тоже могут быть равны нулю, но общее количество ненулевых компонент должно быть достаточным для невырожденности метрики). Поэтому, если полупростая матрица $\boldsymbol{B}$ имеет не более одного собственного вектора с нулевым собственным значением, то при подходящей перенумерации плоских координат, соответствующих однородным функциям $\psi_{i}^{(\alpha)}$, плоская метрика имеет блочно-антидиагональный вид:

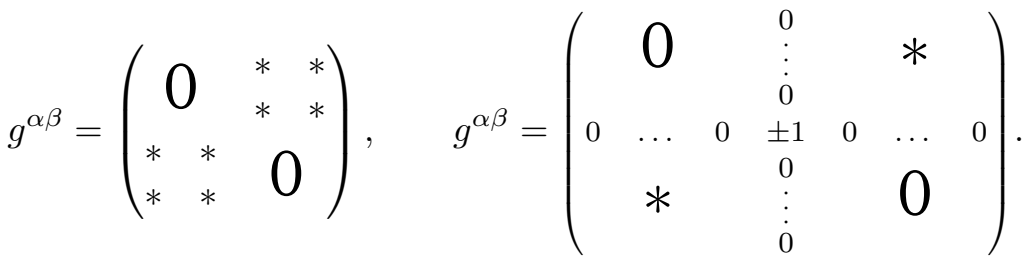

$$
\begin{aligned}
& N=2 n \quad N=2 n+1
\end{aligned}
$$

Мы доказали тем самым следующее утверждение: 
Теорема 7. Если нулевое собственное значение полупростой матриць $\boldsymbol{B}$ имеет кратность не более 1 для плоской однородной егоровской системь координат, то сигнатура (разность числа положительных и отрицательных диагональных коэффициентов в диагональной форме метрики) данной метрики $g^{\alpha \beta}$ минимальна (0 для четного $N u \pm 1$ для нечетного $\left.N\right)$.

\section{§4. Третья гамильтонова структура егоровских однородных систем}

Как мы отмечали выше $(\$ 2)$, рассматриваемые нами физические примеры систем гидродинамического типа обладают в силу условий инвариантности относительно преобразований Галилея и однородности двумя локальными гамильтоновыми структурами.

Из линейной алгебры известно, что кососимметрическая матрица $\boldsymbol{B}$ имеет пары собственных значений, отличающихся знаком. В дальнейшем мы иногда не будем различать собственные значения $c_{\alpha}$ кососимметрической матрицы $\boldsymbol{B}$, отличающиеся знаком.

Разложим квадрат матрицы $\boldsymbol{B}$ следующим образом:

$$
\begin{aligned}
{\left[\boldsymbol{B}^{2}\right]_{i k}=} & \sum_{m \neq i, k}\left(r^{m}-r^{i}\right)\left(r^{m}-r^{k}\right) \beta_{m i} \beta_{m k} \\
= & {\left[\sum_{m \neq i, k}\left(r^{m}\right)^{2} \beta_{m i} \beta_{m k}+\left(r^{i}\right)^{2} \partial_{i} \beta_{i k}+\left(r^{k}\right)^{2} \partial_{k} \beta_{k i}+r^{i} \beta_{i k}+r^{k} \beta_{k i}\right] } \\
& -\left(r^{i}+r^{k}\right)\left[\sum_{m \neq i, k} r^{m} \beta_{m i} \beta_{m k}+r^{i} \partial_{i} \beta_{i k}+r^{k} \partial_{k} \beta_{k i}+\frac{1}{2} \beta_{i k}+\frac{1}{2} \beta_{k i}\right] \\
& +r^{i} r^{k}\left[\sum_{m \neq i, k} \beta_{m i} \beta_{m k}+\partial_{i} \beta_{i k}+\partial_{k} \beta_{k i}\right]+\frac{1}{2}\left(r^{i}-r^{k}\right)\left(\beta_{k i}-\beta_{i k}\right) .
\end{aligned}
$$

Теорема 8. При четном $N=2 n$ однородная егоровская диагональная система гидродинамического типа (2) с плоской метрикой $g_{(1)}^{i i}$ и второй плоской метрикой $g_{(2)}^{i i}=r^{i} g_{(1)}^{i i}$ имеет третью локальную гамильтонову структуру с метрикой $g_{(3)}^{i i}=\left(r^{i}\right)^{2} g_{(1)}^{i i}$, если и только если матрица $\boldsymbol{B}$ невырожденна, полупроста и имеет только одну пару собственных чисел $\pm c$.

ДокАЗАТЕЛЬСтво. В указанном случае $\boldsymbol{B}^{2}=c^{2} E$, где $E$ - единичная матрица, поскольку, применяя $\boldsymbol{B}^{2}$ к любому из ее собственных векторов $\psi_{i}^{(\alpha)}$, получаем $c^{2} \psi_{i}^{(\alpha)}$. С другой стороны, из (39) следует, что при условии наличия двух локальных гамильтоновых структур, заданных метриками $g_{(1)}^{i i}$ и $g_{(2)}^{i i}=r^{i} g_{(1)}^{i i}$ (ср. (10) с $\left.\mu_{i}=r^{i}\right)$,

$$
\left[\boldsymbol{B}^{2}\right]_{i k}=\sum_{m \neq i, k}\left(r^{m}\right)^{2} \beta_{m i} \beta_{m k}+\left(r^{i}\right)^{2} \partial_{i} \beta_{i k}+\left(r^{k}\right)^{2} \partial_{k} \beta_{k i}+r^{i} \beta_{i k}+r^{k} \beta_{k i}=0,
$$

если $i \neq k$, что и означает наличие третьей локальной гамильтоновой структуры (ср. (10) с $\left.\mu_{i}=\left(r^{i}\right)^{2}\right)$.

Обратно, если выполнено условие $(10)$ с $\mu_{i}=\left(r^{i}\right)^{2}$, матрица $\boldsymbol{B}^{2}$ диагональна. В силу нераспадаемости системы $(2) \beta_{i k} \neq 0$ при всех $i \neq k$, и, согласно (17), очевидно, что ни одна из компонент ни одного однородного аннулятора $\psi_{i}^{(\alpha)}$ не 
может быть тождественно равна нулю. Поскольку $\boldsymbol{B}^{2} \psi_{i}^{(\alpha)}=c_{\alpha}^{2} \psi_{i}^{(\alpha)}$, это означает, что диагональная матрица $\boldsymbol{B}^{2}$ имеет одинаковые диагональные коэффициенты $c_{\alpha}^{2}$. Полупростоту матрицы $\boldsymbol{B}$ легко установить, применяя $\boldsymbol{B}^{2}$ к корневым векторам: если $\boldsymbol{B} \vec{v}=c_{\alpha} \vec{v}+\vec{\psi}^{(\alpha)}$, то $\boldsymbol{B}^{2} \vec{v}=c_{\alpha}^{2} \vec{v}+2 c_{\alpha} \vec{\psi}^{(\alpha)} \neq c_{\alpha}^{2} \vec{v}$.

Теорема 9. При нечетном $N=2 n+1$ однородная егоровская диагональная система гидродинамического типа (2) с плоской метрикой $g_{(1)}^{i i}$ и второй плоской метрикой $g_{(2)}^{i i}=r^{i} g_{(1)}^{i i}$ имеет третью нелокальную гамильтонову структуру с метрикой $g_{(3)}^{i i}=\left(r^{i}\right)^{2} g_{(1)}^{i i}$ постоянной кривизны, если и только если матрица $\boldsymbol{B}$ полупроста, имеет однократное собственное число $c_{(0)}=0$ u лишь одну пару ненулевых собственных чисел $c_{( \pm k)}= \pm c(k=1, \ldots, n)$, а сама метрика $g_{(1)}^{\text {ii }}$ имеет степень однородности 0 и зависит только от разностей инвариантов Римана $r^{i}$.

ДокАЗАТЕльство. В этом случае $\left[\boldsymbol{B}^{2}\right]_{i j}=c^{2} \delta_{i j}-\left(c^{2} / g^{00}\right) \psi_{i}^{(0)} \psi_{j}^{(0)}$, где $\psi_{i}^{(0)}-$ базисный элемент ядра матрицы $\boldsymbol{B}, \widehat{R} \psi_{i}^{(0)}=\boldsymbol{B} \psi_{i}^{(0)}=0$, a $g^{00}=\sum_{i} \psi_{i}^{(0)} \psi_{i}^{(0)}-$ соответствующий элемент метрики в плоских координатах. Данное тождество вновь легко проверить, применяя $\boldsymbol{B}^{2}$ к $\psi_{i}^{(\alpha)}$ и учитывая вид (38) метрики в плоских координатах. Снова применяя разложение (39), получаем

$$
\sum_{m \neq i, k}\left(r^{m}\right)^{2} \beta_{m i} \beta_{m k}+\left(r^{i}\right)^{2} \partial_{i} \beta_{i k}+\left(r^{k}\right)^{2} \partial_{k} \beta_{k i}+r^{i} \beta_{i k}+r^{k} \beta_{k i}=4 c^{2} H_{i}^{(0)} H_{k}^{(0)},
$$

так как $\psi_{i}^{(0)}=2 H_{i}^{(0)}$ (ср. $\left.(27)\right)$, что и означает существование третьей, но уже нелокальной гамильтоновой структуры, заданной метрикой $g_{(3)}^{i i}=\left(r^{i}\right)^{2} g_{(1)}^{i i}$ постоянной кривизны $c$, поскольку вычисленные для $g_{(3)}^{i i}$ соотношения $(13)$ имеют вид

$$
\frac{1}{r^{i} r^{k}}\left[\boldsymbol{B}^{2}\right]_{i k}=\frac{4 c^{2}}{r^{i} r^{k}} H_{i}^{(0)} H_{k}^{(0)}, \quad i \neq k .
$$

Наличие соответствующего гамильтониана для данной нелокальной гамильтоновой структуры автоматически вытекает из результатов работы [5].

Обратно, если егоровская диагональная система с двумя плоскими метриками $g_{(1)}^{i i}$ и $g_{(2)}^{i i}=r^{i} g_{(1)}^{i i}$ и однородными $\beta_{i k}$ имеет третью нелокальную гамильтонову структуру с метрикой $g_{(3)}^{i i}=\left(r^{i}\right)^{2} g_{(1)}^{i i}$ постоянной кривизны, то $\left[\boldsymbol{B}^{2}\right]_{i j} /\left(r^{i} r^{k}\right)=$ $d_{i}(r) \delta_{i j}-c \widetilde{H}_{i} \widetilde{H}_{j} /\left(r^{i} r^{k}\right)$, где $\widetilde{H}_{i}(r)=1 / \sqrt{g_{(1)}^{i i}}$, а $d_{i}(r)$ - диагональные элементы некоторые функции. Из однородности коэффициентов $\beta_{i k}$ и их инвариантности относительно сдвига $r^{i} \rightarrow r^{i}+$ const следует, что все произведения $\widetilde{H}_{i} \widetilde{H}_{j}$ имеют степень однородности 0 и инвариантны относительно сдвига; следовательно, выражая сами $\widetilde{H}_{i}$ через эти произведения, получаем, что метрика $g_{(1)}^{i i}$ имеет степень однородности 0 и зависит только от разностей инвариантов Римана $r^{i}$. Применяя матрицу $\boldsymbol{B}^{2}$ к любому ее собственному или корневому вектору, легко выводим равенство всех ее ненулевых собственных чисел и отсутствие корневых векторов.

ТЕорема 10. Однородная егоровская диагональная система гидродинамического типа (2) с плоской метрикой $g_{(1)}^{i i}$ u второй плоской метрикой $g_{(2)}^{i i}=$ 
$r^{i} g_{(1)}^{i i}$ имеет третий нелокальный гамильтонов оператор общего вида (11) с метрикой $g_{(3)}^{i i}=\left(r^{i}\right)^{2} g_{(1)}^{i i}$, если матрица $\boldsymbol{B}$ полупроста.

ДокАЗАТЕЛЬСТво. В этом общем случае

$$
\left[\boldsymbol{B}^{2}\right]_{i j}=c_{1}^{2} \delta_{i j}-\sum_{s} c_{1}^{2} \psi_{i}^{(0, s)} \psi_{j}^{(0, s)}+\sum_{\alpha \neq 1}\left(c_{\alpha}^{2}-c_{1}^{2}\right)\left(\psi_{i}^{(\alpha)} \psi_{j}^{(-\alpha)}+\psi_{i}^{(-\alpha)} \psi_{j}^{(\alpha)}\right),
$$

где аннуляторы $\psi_{i}^{(0, s)}$ - базисные элементы ядра матрицы $\boldsymbol{B}, \psi_{i}^{(\alpha)}-$ ее собственные векторы, $\psi_{i}^{(-\alpha)}-$ ее собственные векторы с противоположными собственными числами, упорядоченные таким образом, что метрика (38) диагональна для $\psi_{i}^{(0, s)}$ и антидиагональна для $\psi_{i}^{(\alpha)}, \psi_{i}^{(-\alpha)}: \sum_{i} \psi_{i}^{(\alpha)} \psi_{i}^{(-\alpha)}=1$, причем мы выбрали одно из собственных значений $c_{1}$ в качестве «основного» диагонального элемента матрицы $\boldsymbol{B}^{2}$. Данное равенство, как и в предыдущих случаях, легко проверить прямой подстановкой в эту формулу собственных векторов матрицы $\boldsymbol{B}$. Это позволяет, используя $(27)$, написать соответствующее разложение (13), гарантирующее наличие гамильтонова оператора (11). В случае, когда $\boldsymbol{B}$ имеет собственные числа $\pm 1 / 2$ (см. ниже $\$ \$ 5,6)$, необходимо выбрать $c_{1}=-1 / 2$ для того, чтобы с помощью $(27)$ выразить необходимые аннуляторы $\psi_{i}^{(\alpha)}$ через соответствующие $H_{i}^{(\alpha)}$.

Заметим, что $H_{i}^{(0)}=\psi_{i}^{(0)} / 2$ могут быть найдены явно без квадратур уже в случае произвольной метрики с однородными $\beta_{i k}$. Например, при $N=3$ получаем $\psi_{i}^{(0)}=r^{j} \beta_{k j}-r^{k} \beta_{j k}$, а при $N=5$ имеем $\psi_{i}^{(0)}=a_{j k} a_{l m}+a_{j l} a_{m k}+a_{j m} a_{k l}$, где $a_{j k}=r^{j} \beta_{k j}-r^{k} \beta_{j k}$. Если метрика егоровская, то при $N=3$ получаем $\psi_{i}^{(0)}=\left(r^{j}-r^{k}\right) \beta_{j k}$, а при $N=5$ имеем $\psi_{i}^{(0)}=\left(r^{j}-r^{k}\right)\left(r^{l}-r^{m}\right) \beta_{j k} \beta_{l m}+$ $\left(r^{j}-r^{l}\right)\left(r^{m}-r^{k}\right) \beta_{j l} \beta_{m k}+\left(r^{j}-r^{m}\right)\left(r^{k}-r^{l}\right) \beta_{j m} \beta_{k l}$. Аналогично выписываются $\psi_{i}^{(0)}$ при $N=2 n-1>5$.

В следующих параграфах мы покажем, как устанавливать наличие вторых и третьих гамильтоновых структур для систем гидродинамического типа, не записанных в инвариантах Римана.

\section{§5. Усредненные $N$-фазные решения уравнения Кортевега-де Фриза и нелинейного уравнения Шрёдингера}

$N$-фазные решения уравнения КдФ были усреднены методом Уизема в [22]. Однако гамильтонов формализм для усредненных уравнений был создан позднее в работах $[1,2,15]$. При усреднении получающиеся системы гидродинамического типа наследуют такие свойства, как галилеева инвариантность, гамильтоновость, однородность. Более того, так как уравнения КдФ галилеево-инвариантно, имеет пару локальных гамильтоновых структур и пару законов сохранения (см. теорему 1, формула (21))

$\partial_{t} u=\partial_{x}\left[u^{2}+\varepsilon^{2} u_{x x}\right], \quad \partial_{t}\left[u^{2}+\varepsilon^{2} u_{x x}\right]=\partial_{x}\left[\frac{4}{3} u^{3}-3 \varepsilon^{2} u_{x}^{2}+2 \varepsilon^{2}\left(u^{2}\right)_{x x}+\varepsilon^{4} u_{x x x x}\right]$,

то после усреднения на $N$-фазном решении полученная $(2 N+1)$-компонентная система гидродинамического типа является также галилеево инвариантной и имеет егоровскую метрику (в силу теоремы 1), зависящую от разностей инвариантов Римана и однородную; следовательно, она имеет пару локальных гамильтоновых 
структур. Аннуляторы первой локальной гамильтоновой структуры усредненного уравнения КдФ состоят из усреднения аннулятора первой локальной гамильтоновой структуры самого КдФ, а также $N$ волновых чисел (из $N$ фаз $\theta_{i}=k_{i} x-\omega_{i} t$ квазипериодического решения) и $N$ соответствующих частных производных по этим волновым числам усредненного лагранжиана. Прямым вычислением было показано, что степень однородности функций $\psi_{i}^{(0)}$, отвечающая усредненному аннулятору первой локальной гамильтоновой структуры, равна 0 , степень однородности функций $\psi_{i}^{(k)}, k=1, \ldots, N$, отвечающая волновым числам, равна $-1 / 2$, степень однородности функций $\psi_{i}^{(N+k)}, k=1, \ldots, N$, отвечающая частным производным по этим волновым числам усредненного лагранжиана, равна $1 / 2$. Все это означает, тем самым (в силу теоремы 9), что усредненные уравнения КдФ имеют третью нелокальную гамильтоновую структуру (наличие первых двух локальных гамильтоновых структур было установлено еще в [2]), ассоциированную с метрикой постоянной кривизны (см. [25]).

Многофазные решения нелинейного уравнения Шрёдингера (НУШ) были усреднены в дипломной работе первого автора; позднее этот результат был опубликован в [6]. Независимо этот результат получен в [21]. Было доказано, что усредненные $N$-фазные решения НУШ имеют три локальные гамильтоновы структуры, а четвертая гамильтонова структура является нелокальной, заданной дифференциально-геометрической скобкой Пуассона с метрикой постоянной кривизны. В работах [1] и [15] был предъявлен вид первых трех гамильтоновых структур в канонической форме - в плоских координатах. Само НУШ, записанное в форме Хасимото, является галилеево инвариантным, имеет три локальные гамильтоновы структуры и пару законов сохранения

$$
\partial_{t}|u|^{2}=i \partial_{x}\left[u \bar{u}_{x}-\bar{u} u_{x}\right], \quad i \partial_{t}\left[u \bar{u}_{x}-\bar{u} u_{x}\right]=\partial_{x}\left[|u|^{4}+4\left|u_{x}\right|^{2}-\left(|u|^{2}\right)_{x x}\right] .
$$

Повторяя все приведенные выше для случая КдФ рассуждения в случае НУШ, также можно показать, что при $N$-фазном усреднении эти свойства наследуются (см. [8]), что является хорошей иллюстрацией к теоремам 1 и 8.

\section{§6. Системы Бенни-Захарова и Яджимы-Оикавы-Мельникова (бездисперсионный предел)}

Система уравнений Бенни (редукция Захарова, см. [4, 16])

$$
u_{t}^{k}=\partial_{x}\left[\frac{\left(u^{k}\right)^{2}}{2}+\sum_{m=1}^{N} \eta^{m}\right], \quad \eta_{t}^{k}=\partial_{x}\left(u^{k} \eta^{k}\right)
$$

галилеево инвариантна $\left(u^{k} \rightarrow u^{k}+c, x \rightarrow x-c t\right)$, имеет пару законов сохранения вида (см. теорему 1, формула (21))

$$
\begin{aligned}
\partial_{t}\left(\sum_{m=1}^{N} \eta^{m}\right) & =\partial_{x}\left(\sum_{m=1}^{N} u^{m} \eta^{m}\right) \\
\partial_{t}\left(\sum_{m=1}^{N} u^{m} \eta^{m}\right) & =\partial_{x}\left[\sum_{m=1}^{N}\left(u^{m}\right)^{2} \eta^{m}+\frac{1}{2}\left(\sum_{m=1}^{N} \eta^{m}\right)^{2}\right]
\end{aligned}
$$


и первую локальную гамильтонову структуру

$$
u_{t}^{k}=\partial_{x} \frac{\delta H}{\delta \eta^{k}}, \quad \eta_{t}^{k}=\partial_{x} \frac{\delta H}{\delta u^{k}},
$$

заданную однородной, зависящей от разностей инвариантов Римана егоровской метрикой (в инвариантах Римана $\lambda^{k}, k=1, \ldots, 2 N$, см. [13])

$$
g^{i i}=\sum_{m=1}^{N} \frac{\eta^{m}}{\left(\mu^{i}+u^{m}\right)^{3}},
$$

где характеристические скорости $\mu^{i}$ и инварианты Римана $\lambda^{i}$ находятся из системы (см. [23])

$$
\lambda^{i}=\mu^{i}+\sum_{m=1}^{N} \frac{\eta^{m}}{\mu^{i}+u^{m}}, \quad 1=\sum_{m=1}^{N} \frac{\eta^{m}}{\left(\mu^{i}+u^{m}\right)^{2}} .
$$

Из сравнения степеней однородности плоских координат $\left(u^{k}, \eta^{k}\right)$ и метрики $g^{i i}$ видно, что степень однородности функций $\psi_{i}^{(k)}, k=1, \ldots, N$, отвечающая первой половине плоских координат $u^{k}$, равна $-1 / 2$, а степень однородности функций $\psi_{i}^{(N+k)}, k=1, \ldots, N$, отвечающая второй половине плоских координат $\eta^{k}$, равна $1 / 2$. Отсюда в силу теорем 1 и 8 следует, что система Бенни-Захарова (40) имеет также вторую и третью локальные гамильтоновы структуры (см. $[9,10])$.

Система Яджимы-Оикавы (так называемый длинно-короткий резонанс), обобщенная на $N$-компонентный случай (система Мельникова-I, см. [26]), в бездисперсионном пределе имеет вид

$$
u_{t}^{k}=\partial_{x}\left[\frac{\left(u^{k}\right)^{2}}{2}+w\right], \quad \eta_{t}^{k}=\partial_{x}\left(u^{k} \eta^{k}\right), \quad w_{t}=\partial_{x}\left[\sum_{m=1}^{N} \eta^{m}\right] .
$$

Она также галилеево инвариантна $(w \rightarrow w+c, x \rightarrow x-c t)$, имеет пару законов сохранения требуемого в теореме 1 вида

$$
\partial_{t} w=\partial_{x}\left(\sum_{m=1}^{N} \eta^{m}\right), \quad \partial_{t}\left(\sum_{m=1}^{N} \eta^{m}\right)=\partial_{x}\left[\sum_{m=1}^{N} u^{m} \eta^{m}\right]
$$

и первую локальную гамильтонову структуру

$$
u_{t}^{k}=\partial_{x} \frac{\delta H}{\delta \eta^{k}}, \quad \eta_{t}^{k}=\partial_{x} \frac{\delta H}{\delta u^{k}}, \quad w_{t}=\partial_{x} \frac{\delta H}{\delta w},
$$

заданную однородной, зависящей от разностей инвариантов Римана егоровской метрикой (в инвариантах Римана $\lambda^{k}, k=1, \ldots, 2 N+1$ )

$$
g^{i i}=1-2 \sum_{m=1}^{N} \frac{\eta^{m}}{\left(\mu_{i}+u^{m}\right)^{3}}
$$

где характеристические скорости $\mu_{i}$ и инварианты Римана $\lambda_{i}$ находятся из системы

$$
\lambda^{i}=\frac{\mu_{i}^{2}}{2}+w-\sum_{m=1}^{N} \frac{\eta^{m}}{\mu_{i}+u^{m}}, \quad \mu_{i}+\sum_{m=1}^{N} \frac{\eta^{m}}{\left(\mu_{i}+u^{m}\right)^{2}}=0 .
$$


Из сравнения степеней однородности плоских координат $\left(w, u^{k}, \eta^{k}\right)$ и метрики $g^{i i}$ видно, что степень однородности функций $\psi_{i}^{(0)}$, отвечающая плоской координате $w$, равна 0 , степень однородности функций $\psi_{i}^{(k)}, k=1, \ldots, N$, отвечающая первой половине плоских координат $u^{k}$, равна $-1 / 2$, а степень однородности функций $\psi_{i}^{(N+k)}, k=1, \ldots, N$, отвечающая второй половине плоских координат $\eta^{k}$, равна $1 / 2$. Отсюда в силу теорем 1 и 9 следует, что система ЯОМ имеет также вторую (локальную) и третью (нелокальную, с метрикой постоянной кривизны) гамильтоновые структуры.

Благодарности. Авторы выражают искреннюю признательность Е. В. Ферапонтову за многочисленные и стимулирующие обсуждения, живой интерес и удачные советы, В. Л. Алексееву за комментарии по методу Уизема и алгеброгеометрическому подходу к усреднению $N$-фазных решений уравнения КдФ и НУШ, а также нашему учителю Сергею Петровичу Новикову за интерес к работе и замечания.

\section{ЛИТЕРАТУРА}

1. Алексеев В. Л. О нелокальных гамильтоновых операторах гидродинамического типа, связанных с уравнениями Уизема. УМН, 50, вып. 6, 165-166 (1995).

2. Дубровин Б. А., Новиков С. П. Гамильтонов формализм одномерных систем гидродинамического типа и метод усреднения Боголюбова-Уизема. Докл. АН СССР, 270, № 4, 781-785 (1993).

3. Егоров Д. Ф. Работы по дифференциальной геометрии. Наука, М., 1970.

4. Захаров B. E. Уравнения Бенни и квазиклассическое приближение в методе обратной задачи. Функц. анализ и его прил., 14, вып. 2, 15-24 (1980).

5. Мохов О. И., Ферапонтов $E$. В. Нелокальные гамильтоновы операторы гидродинамического типа, ассоциированные с метриками постоянной кривизны. УМН, 45, вып. 3, 191-192 (1993).

6. Павлов М. В. Нелинейное уравнение Шрёдингера и метод усреднения БоголюбоваУизема. Теор. матем. физ., 71, вып. 3, 351-356 (1987).

7. Павлов М. В. Эллиптические координаты и мультигамильтоновы структуры систем гидродинамического типа. Докл. РАН, 339, вып. 1, 21-23 (1994).

8. Павлов М. В. Мультигамильтоновы структуры уравнений Уизема. Докл. РАН, 338, № 2, 165-167 (1994).

9. Павлов М. В. О локальных гамильтоновых структурах системы Бенни. Докл. РАН, 338, № 1, 33-34 (1994).

10. Павлов М. В., Царев С. П. О законах сохранения уравнений Бенни. УМН, 46, вып. 4, 169-170 (1991).

11. Ферапонтов E. B. Дифференциальная геометрия нелокальных гамильтоновых операторов гидродинамического типа. Функц. анализ и его прил., 25, вып. 3, 37-49 (1991).

12. Царев С. П. О скобках Пуассона и одномерных гамильтоновых системах гидродинамического типа. Докл. АН СССР, 282, № 3, 534-537 (1985).

13. Царев С. П. Геометрия гамильтоновых систем гидродинамического типа. Обобщенный метод годографа. Изв. АН СССР. сер. матем., 54, № 5, 1048-1068 (1990).

14. Царев С. П. Дифференциально-геометрические методы интегрирования систем гидродинамического типа. Дисс. д. ф.-м.н., М., 1993.

15. Alekseev V. L., Pavlov M. V. Hamiltonian structures of the Whitham equations. In: Proceedings of the Conference on NLS, Chernogolovka, 1994.

16. Benney D. J. Some properties of long nonlinear waves. Stud. Appl. Math., 52, 45-50 (1973). 
17. Darboux G. Leçons sur les systèmes orthogonaux et les coordonnées curvilignes. Gautier-Villar, Paris, 1910.

18. Dubrovin B. A. Geometry of 2D topological field theories. In: Integrable Systems and Quantum Groups (Montecatini, Terme, 1993), M. Francaviglia, S. Greco, eds.. Lecture Notes in Math., Vol. 1620, 120-348 (1996).

19. Dubrovin B. A. Integrable systems and classification of $2 D$ topological field theories. In: Integrable Systems, The J.-L. Verdier Memorial Conference, Actes du Colloque International de Luminy (O. Babelon, P. Cartier, Y. Kosmann-Schwarzbach, eds.), Birkhäuser, 1993, pp. 313-359.

20. Ferapontov E. V., Pavlov M. V. Quasiclassical limit of Coupled KdV equations. Riemann invariants and multi-Hamiltonian structure. Phys. D, 52, 211-219 (1991).

21. Forest M. G., Lee J. E. Geometry and modulation theory for the periodic nonlinear Schrödinger equation. In: Oscillation Theory, Computation, and Methods of Compensated Compactness (C. Dafermos et al., eds.), Vol. 2, IMA, Springer-Verlag, New York, 1986.

22. Flaschka H., Forest M. G., McLaughlin D. W. Multiphase averaging and the inverse spectral solution of the Korteweg-de Vries equation. Comm. Pure Appl. Math., 33, 739-784 (1980).

23. Gibbons $J$. Collisionless Boltzmann equations and integrable moment equations. Phys. D, 3, No. 3, 503-511 (1981).

24. Gumral H., Nutku Y. Multi-Hamiltonian structure of equations of hydrodynamic type. J. Math. Phys., 31, No. 11, 2606-2611 (1990).

25. Pavlov M. V. Integrable systems and metrics of constant curvature. J. Nonlinear Math. Phys., 9, Suppl. 1, 173-191 (2002).

26. Oevel W., Sidorenko J., Strampp W. Hamiltonian structures of Melnikov system and its reductions. Inverse Problems, 9, 737-747 (1993).

Красноярский государственный педагогический университет, e-mail: tsarev@edk.krasnoyarsk.su 